\title{
ESTUDIO DE DOS RASAS INTERMAREALES AL NORTE DE TENERIFE COMO ZONA DE CRIADERO DE JUVENILES DE EPINEPHELUS MARGINATUS (LOWE, 1834)
}

\author{
Sergio Moreno-Borges*, Carlos Aspiroz, \\ Sandra Hernández-Mingorance, Marina Jaramillo-Delgado, \\ Manuel A. Marrero \& Mario Ortiz-López
}

\section{RESUMEN}

Se realizó una serie de muestreos en el intermareal rocoso al norte de la isla de Tenerife (Islas Canarias, España) con el objetivo de recopilar datos de abundancia y tallas de la especie Epinephelus marginatus (Lowe, 1834), familia Serranidae, conocida coloquialmente como mero. Esta especie es un recurso pesquero importante con una amplia distribución en zonas litorales, y cuyas zonas de cría son los charcos intermareales. En el presente estudio se muestrearon charcos al azar de diferentes volúmenes: pequeños $\left(<10 \mathrm{~m}^{3}\right)$, medianos $\left(10 \mathrm{~m}^{3}-\right.$ $\left.80 \mathrm{~m}^{3}\right)$ y grandes $\left(>80 \mathrm{~m}^{3}\right)$, con la finalidad de encontrar diferencias en la abundancia y la talla en función del tamaño del charco. Las zonas de estudio se localizaron en Punta del Hidalgo y Finca El Apio, tomando datos en 22 charcos en los cuales se contaron un total de ocho juveniles de E. marginatus. Los análisis mostraron una influencia significativa del tamaño del charco, de tal forma que los individuos de mayor talla se encontraron en los charcos de mayor volumen.

Palabras Clave: charcos intermareales, Epinephelus marginatus, zona de cría.

STUDY OF TWO INTERTIDAL BENCHS AT THE NORTH COAST OF TENERIFE AS NURSERY AREA FOR THE JUVENILES OF EPINEPHELUS MARGINATUS (LOWE, 1834)

\begin{abstract}
A set of samplings were carried out at the rocky intertidal shore of the north of Tenerife island (Canary Islands, Spain) with the objective of compile abundance and size data from the species Epinephelus marginatus (Lowe, 1834) belonging to Serranidae family, known as dusky groupers in colloquial language. This species is an important fishing resource with a wide distribution along the rocky shores, observing juvenile populations inhabiting intertidal pools. In the present research a set of intertidal pools were sampled randomly in function of their size: smalls $\left(<10 \mathrm{~m}^{3}\right)$, medium $\left(10 \mathrm{~m}^{3}-80 \mathrm{~m}^{3}\right)$ and large $\left(>80 \mathrm{~m}^{3}\right)$ with the purpose of determine if there is an effect of this factor over the abundance and size values observed. Study area was located in Punta del Hidalgo and Finca El Apio, recording data from 22 tidalpools with a total number of 8 juveniles of $E$. marginatus. The statistical analysis showed a significant effect of the size of the pools, finding the biggest individuals at the biggest pools.
\end{abstract}

KEYworDs: intertidal pools, Epinephelus marginatus, nursery zones.

DOI: http://doi.org/10.25145/j.SI.2018.01.009

Revista Scientia Insularum, 1; diciembre 2018, pp. 141-148; ISSN: e-2659-6644 
Epinephelus marginatus es un teleósteo de la familia Serranidae que habitan el litoral rocoso y cuevas submarinas, desde el intermareal hasta los 250-300 m de profundidad (Bruslé 1985; Tortonese 1986; Heemstra $y$ Randall 1993). Con frecuencia los juveniles son observados en los charcos intermareales, donde se refugian durante sus etapas más tempranas de vida hasta que son capaces de sobrevivir en hábitats más expuestos a la depredación (Azevedo et al. 1995; Andrade et al. 2003; Lobato et al. 2016). Su área geográfica de distribución comprende el Atlántico Este y Suroeste, la costa oeste del océano Índico, el mar Mediterráneo y el mar Negro (Heemstra y Randall 1993; Cornish y Harmelin-Vivien 2004; Irigoyen et al. 2005). Son de crecimiento muy lento y tardan en torno a cinco ańos en alcanzar la madurez sexual. Son hermafroditas protóginos monoándricos, es decir, los juveniles primero desarrollan órganos sexuales femeninos (Chauvet 1988; Fennessy 2006), y posteriormente sufren un cambio de sexo. Este fenómeno está regulado por procesos fisiológicos muy sensibles y puede verse alterado por la influencia de multitud de factores como pueden ser la sobrepesca, reduciendo el número de reproductores de las poblaciones y obligando a los individuos más jóvenes a madurar en una etapa más temprana, o por cambios físico-químicos de las condiciones del medio que alteran los procesos fisiológicos de estos animales (Reńones et al. 2010; García et al. 2013).

El mero es considerado un importante recurso pesquero en prácticamente todos los territorios en los que se distribuye y como consecuencia sus poblaciones están sufriendo sobrepesca (Begossi et al. 2012). Por estas razones ha sido incluido en la lista roja de especies amenazadas de la IUCN (Cornish y Harmelin-Vivien 2004).

En las costas de la vertiente norte de Tenerife es frecuente encontrar plataformas rocosas, donde se forman charcos o piscinas naturales con la bajamar, las cuales actúan como ecosistemas ideales para el desarrollo de multitud de especies marinas, entre las que se ha observado en múltiples ocasiones la presencia de Epinephelus marginatus. Las localidades de la Punta del Hidalgo y la Finca El Apio son dos localidades de Tenerife que reúnen estas condiciones (Alfonso et al. 2015), por ello las convierte en zonas de un gran interés para la realización de estudios ecológicos como el presente. En este artículo pretendemos analizar la abundancia y talla de meros de la especie $E$. marginatus en los charcos de estas dos localidades, con el fin de comprobar la influencia de la localidad y el tamaño del charco en los parámetros a estudio.

El presente estudio se llevó a cabo en los días 10, 11 y 12 de febrero de 2016, coincidiendo siempre con la marea baja matutina. Se realizó en dos localidades de la vertiente norte de la isla de Tenerife, concretamente en la Punta del Hidalgo y Finca El Apio, las cuales se separan por una distancia de unos $9 \mathrm{~km}$ por la costa (figura 1).

Los muestreos se realizaron por medio de la inspección visual de los charcos, con la ayuda de equipo ligero de buceo, buscando la presencia de estos peces en los huecos y oquedades de los charcos, ya que, por su comportamiento, estos son los

* Máster de Biología Marina: Biodiversidad y Conservación. Universidad de La Laguna. Tenerife, Islas Canarias, España. Autor para la correspondencia: sergiomb93@hotmail.com. 


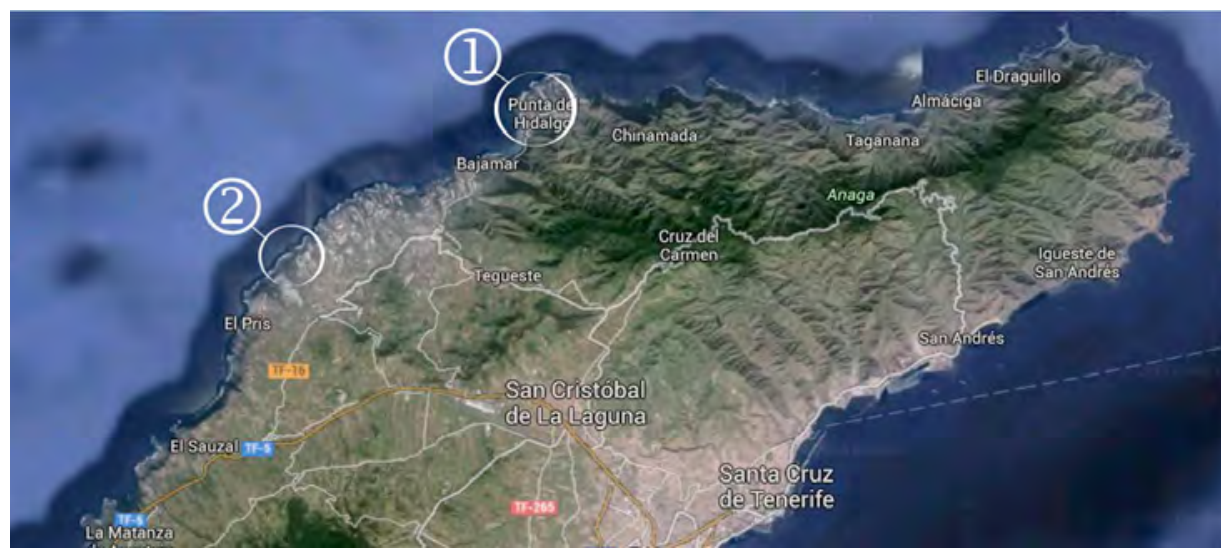

Figura 1. Localización de las dos zonas de muestreo en Tenerife.

1. Punta del Hidalgo $\left(28^{\circ} 34{ }^{\prime} 28.4 » \mathrm{~N} 16^{\circ} 19^{\prime} 49.4 » \mathrm{~W}\right)$;

2. Finca El Apio $\left(28^{\circ} 31^{\prime} 38.5 » \mathrm{~N} 16^{\circ} 24^{\prime} 36.9 » \mathrm{~W}\right)$.

lugares que suelen elegir como refugio. Así mismo, se estimó visualmente la talla de los ejemplares encontrados, en centímetros, empleando como referencia una tablilla previamente medida.

Se muestrearon un total de 22 charcos que fueron seleccionados en función de su volumen. Este valor fue estimado posteriormente gracias a la fórmula del volumen de la semielipsoide (Volumen $=\left(4 / 3 \pi r_{1} r_{2} r_{3}\right) / 2$, donde las $r_{i}$ corresponden con los radios a lo ancho, a lo largo y la profundidad del charco). En función de este volumen se realizó una clasificación de los charcos, considerándolos pequeños si su volumen era inferior a $10 \mathrm{~m}^{3}$, medianos, si este se comprendía entre los $10 \mathrm{~m}^{3}$ y los $80 \mathrm{~m}^{3}$ y por último grandes, si superan los $80 \mathrm{~m}^{3}$.

De los 22 charcos muestreados, 14 son de la Punta del Hidalgo, mientras que ocho pertenecen a Finca El Apio.

Se realizó un análisis de la varianza univariante por permutaciones (PERANOVA) con un diseño de dos vías, tomando como factor fijo el tamaño de los charcos (con tres niveles: pequeño, mediano, grande), y como factor aleatorio la localidad (Anderson 2001, 2004).

A continuación, se realizó una matriz triangular en base a las distancias euclídeas, para cada una de las variables: abundancia y talla media. No se aplicó ningún tipo de transformación a los datos, y para aquellos factores que resultaron significativos se realizaron comparaciones por pares a posteriori. El análisis se llevó a cabo estableciendo un nivel de significación de $\alpha=0,05$ (Anderson 2004). Los datos se analizaron mediante el paquete estadístico PRIMER 6 y PERMANOVA + v.1.0.1.

En los muestreos se observaron un total de ocho meros distribuidos entre los tres tipos de charcos y las dos localidades, tal como se observa en la tabla 1. De acuerdo con el ANOVA, no existen diferencias significativas en la abundancia 
respecto al factor «Tamaño de charco» (Pseudo-F= 4,83; $\mathrm{p}=0,146)$ ni el factor «Localidad» (Pseudo-F=0,28; $\mathrm{p}=0,627$ ).

\begin{tabular}{|c|c|c|c|c|c|}
\hline \multicolumn{6}{|c|}{$\begin{array}{l}\text { TABLA 1. DISTRIBUCIÓN DE LOS INDIVIDUOS OBSERVADOS. TAMAÑO DEL CHARCO, } \\
\text { LOCALIDAD, NÚMERO DE CHARCOS ESTUDIADOS, VOLUMEN DE LOS CHARCOS }\left(\mathrm{m}^{3}\right) \text {, } \\
\text { NÚMERO DE INDIVIDUOS ENCONTRADOS Y TALLA MEDIA DE LOS MISMOS }(\mathrm{cm})\end{array}$} \\
\hline Tamaño & LOCALIDAD & NÚMERo & Volumen & N. ${ }^{\circ}$ INDIVIDUOS & TAlla \\
\hline \multirow{2}{*}{ Pequeños } & Punta del Hidalgo & 7 & 36,50 & 1 & 5 \\
\hline & Finca El Apio & 3 & 21,44 & 1 & 7 \\
\hline \multirow{2}{*}{ Medianos } & Punta del Hidalgo & 4 & 175,71 & 0 & 0 \\
\hline & Finca El Apio & 2 & 57,88 & 0 & 0 \\
\hline \multirow{2}{*}{ Grandes } & Punta del Hidalgo & 3 & 564,44 & 4 & 6,5 \\
\hline & Finca El Apio & 3 & 531,38 & 2 & 9 \\
\hline
\end{tabular}

En cuanto a la talla, los meros observados fueron individuos juveniles que oscilaron entre los 5 y los $10 \mathrm{~cm} \mathrm{y,} \mathrm{de} \mathrm{acuerdo} \mathrm{con} \mathrm{el} \mathrm{análisis} \mathrm{ANOVA} \mathrm{por} \mathrm{permu-}$ taciones, presentaron diferencias significativas en función del tamaño del charco (Pseudo- $\mathrm{F}=23,95 ; \mathrm{p}=0,037$ ), existiendo diferencias entre los charcos pequeños y los grandes $(t=18,25 ; p=0,045)$, pero no entre los medianos con los grandes $(t=5,00$; $\mathrm{p}=0,11)$, ni con los pequeños $(\mathrm{t}=1,88 ; \mathrm{p}=0,285)$. El factor localidad no fue significativo (Pseudo-F=0,81; $\mathrm{p}=0,389$ ) respecto a la talla.

El estudio pone de manifiesto la existencia de una relación entre la talla de los individuos de Epinephelus marginatus y el tamaño del charco. Los individuos de tallas mayores se localizaron en charcos por encima de $80 \mathrm{~m}^{3}$ y los individuos de tallas más pequeñas están presentes en los charcos con menos de $10 \mathrm{~m}^{3}$, apreciando que los charcos entre 10 y $80 \mathrm{~m}^{3}$ (clasificado como tamaño medio) no muestran diferencias entre lo encontrado en ellos y lo que hay en los otros dos grupos de charcos (pequeños y grandes). Este resultado podría indicar una preferencia de los meros por charcos más amplios a medida que crecen de tamańo, una posible explicación para esto sería que los meros en etapas más tempranas son más vulnerables a los depredadores debido a su reducido tamaño, viéndose obligados a ocupar charcos de menores tamaños que ofrecen una mayor protección frente a los depredadores potenciales (como pueden ser otros peces de mayor tamaño y pulpos), a medida que los individuos van creciendo podrán ocupar charcos de mayor volumen, hasta que finalmente salgan a mar abierto. Del mismo modo, sería interesante tomar más datos para verificar el efecto de las variables ambientales en la segregación por tallas.

Azevedo y colaboradores (1995) reportaron datos de juveniles de Epinephelus marginatus que habitaban en una piscina intermareal de gran tamaño en Azores (aproximadamente $3500 \mathrm{~m}^{3}$ ). En este estudio encontraron individuos con tallas desde los 4 hasta los $36 \mathrm{~cm}$. Estos autores sugirieron una diferenciación de los individuos por clases de edad en base a los anillos que presentaban las escamas bajo la aleta pectoral izquierda, obteniendo un grupo con individuos entre 4-7 cm de talla sin ningún anillo; otro con individuos de $13-17 \mathrm{~cm}$ con un anillo; de $18-25 \mathrm{~cm}$ presentaban 1-2 anillos; mientras en el único individuo de 36 se contaron al menos cuatro 
anillos. En otros estudios, los rangos de tallas que se observaron fueron bastante variables, Bertoncini et al. (1999) encontró tallas de 2 a $3 \mathrm{~cm}$, mientras que Andrade et al. (2003) observaron individuos desde 2,2 cm hasta 14,6 cm. Estos últimos no especifican el tamaño de los charcos muestreados, aunque lo más probable es que se trate de charcos más similares a los muestreados en el presente estudio, mientras que la piscina natural estudiada por Azevedo et al. (1995) es muy superior en volumen y probablemente es por ello que encontraron individuos de 4-7 cm, conviviendo con otros de tallas superiores a $20 \mathrm{~cm}$. En términos generales, puesto que según los resultados de este estudio existen evidencias de que el tamaño de los charcos tiene un efecto en la distribución de los juveniles de E. marginatus, se podría esperar que en este tipo de zonas intermareales, los individuos ocupen charcos de mayor volumen a medida que crecen en talla, hasta que finalmente los individuos preadultos salen a mar abierto y ocupan las aguas someras del litoral. En estudios futuros sería interesante diseñar los muestreos para analizar los rangos de tallas a lo largo de un ańo entero y comprobar si se cumple la existencia de otros grupos de edad.

Por otro lado, al tratar de verificar la existencia de una relación entre la abundancia de la especie y el tamaño de los charcos y la localidad, no se pudo comprobar que estos factores influyan en la variable abundancia. Es interesante resaltar que esta especie presenta comportamientos territoriales (Zabala et al. 1997), observándose que los individuos pasan la mayor parte del tiempo dispersos entre sí, formando grandes congregaciones solo en la época de reproducción en verano. Es posible que la explicación a que no exista relación entre la abundancia de los juveniles de meros y el tamaño de los charcos sea también que mantienen este comportamiento territorial ya desde etapas tempranas.

En cuanto al comportamiento reproductivo de Epinephelus marginatus, se sabe que esta etapa ocurre durante finales de primavera y todo el verano y a continuación se produce una dispersión espacial de los reproductores, especialmente en meses de invierno (Reñones et al. 2010). Los muestreos se realizaron durante un único periodo estacional, en febrero de 2016; futuras investigaciones podrían centrarse en realizar muestreos teniendo en cuenta el factor estacional, realizándose en los mismos charcos, pero en diferentes estaciones a lo largo de uno o dos ańos. Esto sería de una gran utilidad para conocer mejor el desarrollo de los juveniles de meros en toda la zona del intermareal.

En términos generales, este estudio pone de manifiesto que las zonas intermareales de Canarias actúan como zonas de cría de esta especie tan emblemática, y que existe también proceso de segregación por tallas de los individuos en función del tamaño del charco. 


\section{AGRADECIMIENTOS}

Este estudio se realizó en el contexto del Máster de Biología Marina de la Universidad de La Laguna, curso 2015-2016. Agradecemos la ayuda y colaboración prestada por el profesor José Carlos Hernández.

\section{CONTRIBUCIÓN DE LOS AUTORES}

Conceptualización: SM.

Metodología y trabajo de campo: SM, CA, SHM, MJD, MAM, MOL. Análisis de datos: SM con la ayuda del profesor José Carlos Hernández. Preparación del escrito original: SM, CA, SHM, MJD, MAM, MOL.

Corrección y edición del escrito definitivo: SM, CA, SHM, MJD, MAM, MOL. 


\section{REFERENCIAS}

Alfonso, B., Sarabia, A., Sancibrián, I., Alfaro, A., Adern, N. y Hernández, J.C. 2015. Efecto de la actividad humana sobre la distribución y estructura poblacional del burgado Phorcus sauciatus (Koch, 1845). Rev. Acad. Canar. Cien. 27: 333-343.

Anderson, M.J. 2001. A new method for non-parametric multivariate analysis of variance. Austral Ecol. 26: 32-46.

ANDERson, M.J. 2004. PERMANOVA_2factor: a FORTRAN computer program for permutational multivariate analysis of variance (for any two-factor ANOVA design) using permutation tests. Department of Statistics, University of Auckland. New Zealand.

Andrade, Á., Machado, L., Hostim-Silva, M. y Barreiros, J. 2003. Reproductive Biology of the Dusky Grouper Epinephelus marginatus (LOWE, 1834). Braz. Arch. Biol. Technol. 46: 373-381.

Azevedo, J.M.N., Rodrigues, J.B., Mendizabal, M. y Arruda, L.M. 1995. Study of a sample of dusky groupers, Epinephelus marginatus (Lowe, 1834), caught in a tide pool at Lajes do Pico, Azores. Bol. Mus. Munic. Funchal. 4: 55-64.

Begossi, A., Salyvonchyк, S., Barreto, T., Nora, V., Lopes, P.F.M. y Silvano, R.A.M. 2012. Small-scale fisheries and conservation of drusky grouper (Garoupa), Epinephelus marginatus (Lowe, 1834) in the southeastern Brazilian coast. Sci. J. Agric. Res. Manag. 2012: 1-4.

Bertoncini, A.A., Barreiros, J.P., Hostim-Silva, M. e Inez, V.M. (1999). Ictiofauna ocorrente em sistemas intertidais no município de Penha-SC. In: Resumos do XIII Encontro Brasileiro de Ictiologia. Universidade Federal de São Carlos- SP. 209 pp.

Bruslé, J. 1985. Expose synoptique des donnés biologiques sur les mérous Epinephelus aeneus (Geoffroy Saint Hilaire, 1809) et Epinephelus guaza (Linnaeus, 1758) de l'océan Atlantique et de le Méditerranée. FAO Synopsis sur les pêches. 129: 1-64.

Chauvet, C. 1988. Etude de la croissance du mérou Epinephelus guaza (Linné, 1758) des côtes tunisiennes. Aquat. Living. Resour. 1: 277-288.

Cornish, A. y Harmelin-Vivien, M. (Grouper y Wrasse Specialist Group). 2004. Epinephelus marginatus. The IUCN Red List of Threatened Species 2004: e.T7859A12857009. Downloaded on 28 February 2016.

Fennessy, ST. 2006. Reproductive biology and growth of the yellow belly rock cod Epinephelus marginatus (Serranidae) from South-East Africa. Afr. J. Mar. Sci. 28: 1-11.

Garcia, C.E., Araújo, B.C., Mello, P.H., Narcizo, A.M., Rodrigues-Filho, J.A., MedraDo, A.T., Zampieri, R.A., Floeter-Winter, L.M. y Moreira, R.G. 2013. Involvement of pituitary gonadotropins, gonadal steroids and breeding season in sex change of protogynous dusky grouper, Epinephelus marginatus (Teleostei: Serranidae), induced by a non-steroidal aromatase inhibitor. Gen. Comp. Endocrinol. 192: 170-180.

Heemstra, P.C. y Randall, J.E. 1993. Groupers of the world (Family Serranidae, Subfamily Epinephelidae). An annotated and illustrated catalogue of the grouper, rockcod, hind, coral grouper and lyretail species known. FAO Species catalogue. FAO Fisheries Synopsis. 16: $1-382$.

Irigoyen, A.J., Galva, D.E. y Venerus, L.A. 2005. Occurrence of dusky grouper Epinephelus marginatus (Lowe, 1834) in gulfs of northern Patagonia, Argentina. J. Fish. Biol. 67: 1741-1745. 
Lobato, C.M.C., Soares B.E., Begot T.O.R. y DeAssisMontag, L.F. 2016. Tidal pools as habitat for juveniles of the goliath grouper Epinephelus itajara (Lichtenstein 1822) in the Amazonian coastal zone, Brazil. Nat. Conservação. 14(1), 20-23.

Reñones, O., Grau, A., Mas, X., Riera, F. y Saborido-Rey, F. 2010. Reproductive pattern of an exploited dusky grouper Epinephelus marginatus (Lowe 1834) (Pisces: Serranidae) population in the western Mediterranean. Sci. Mar. 74:523-537.

Tortonese, E. 1986. Serranidae. In Whitehead, P.J.P; Bauchot, M.L.; Hureau, J.C.; Nielsen J. and Tortonese, E. (eds.). Fishes of the North Atlantic and Mediterranean. FAO, 2: 780-792.

Zabala, M., Louisy, P., García-Rubies, A. y Gracia, V. 1997. Socio-behavioural context of reproduction in the Mediterranean dusky grouper Epinephelus marginatus (Lowe, 1834) (Pisces: Serranidae) in the Medes Islands Marine Reserve (NW Mediterranean, Spain). Sci. Mar. 61: 79-89. 